\title{
Le Pilotage De La Performance Des Fournisseurs Par Les Pratiques De Gestions De Chaine Logistique (Supply Chain Management) Et L'apport De L'audit Combine «Qualité-Logistique» «Cas De Renault Tanger»
}

\author{
Fouad Moutaoukil, (Doctorant en Sciences de Gestion) \\ Mohamed Amine M'barki, (Professeur de l'enseignement \\ Supérieur) \\ Souad Boungab, (Enseignant chercheur) \\ Groupe de Recherche « Management et Systèmes d'information » GREMSI \\ Ecole Nationale de Commerce et de Gestion de Tanger ENCGT, Tanger, \\ Morocco
}

Doi:10.19044/esj.2018.v14n16p117 URL:http://dx.doi.org/10.19044/esj.2018.v14n16p117

\section{Abstract}

Based on the current economic environment of companies, with the focus on core business and the increasing use of outsourcing, a good relationship management with suppliers is very crucial to increase the performance of the companies.

Since the rate of purchases in the value added of the product keeps increasing, suppliers who are considered as partners have a significant role to play. This situation poses the problem of managing the customer-supplier relationship because a greater part of the performance depends on suppliers and the service providers.

Due to these observations, there is the need for companies to secure their supply chain, especially in terms of QCD (Quality, Cost and Delay). The control of these three elements is a solid asset that is used to remain competitive and to keep their strategic stake in the logistics chain.

The SCM practices related to supplier management are defined as a set of activities within an organization to ensure an effective supply chain management. This paper focuses on exploring the best practices in term of supplier management used to ensure high performance in the automotive sector in Morocco.

In other words, the objective of this research is to assess the actual state and the perspectives for applying a security approach and an appraisal of the 
suppliers' performance, based on management practices and a combination of quality and logistics audits.

This research contributes to the study of the link between the SCM practices and the performance based on a qualitative approach applied with a business case within the automobile manufacturer Renault Tanger in Morocco.

Keywords: Customer-Supplier Relationship, SCM practices, Combined audit, Quality, Logistics, management, performance

\section{Resume}

Dans l'environnement économique actuel des entreprises, avec le recentrage sur le métier de base et le recours croissant à l'externalisation des activités, une bonne gestion de la relation avec les fournisseurs constitue un vrai levier d'accroissement de la performance. En effet, le poids des achats dans la valeur ajoutée du produit ne cesse pas d'augmenter ; les fournisseurs jouent un rôle stratégique et sont considérés comme des partenaires. Cette situation pose le problème du pilotage de la relation client-fournisseur car une grande partie de la performance se trouve entre les mains des fournisseurs et des prestataires. De ces constats, découle pour les entreprises un réel besoin de sécuriser la Supply Chain, surtout en matière du triplet QCD (Qualité, Coût et Délai). La maîtrise de ces trois éléments est un atout solide pour rester compétitif et un enjeu stratégique fort dans la chaine logistique. Dans cette étude, on essaie d'explorer les meilleures pratiques de gestion des fournisseurs utilisées pour assurer la performance des approvisionnements dans le secteur automobile au Maroc. Concrètement, l'objectif de cette recherche est de mettre le point sur l'état des lieux et les perspectives d'application d'une démarche de sécurisation et d'évaluation de la performance des fournisseurs, axée sur les pratiques de gestion et basée sur la combinaison des audits qualité et logistique. Cette recherche prétend contribuer à l'étude de lien entre les pratiques de SCM et la performance en s'appuyant sur une méthode qualitative sous forme d'une étude de cas unique au sein du constructeur automobile Renault Tanger au Maroc.

Mots-clés : Relation client-fournisseur, Pratiques de SCM, Audit combiné, Pilotage, Performance

\section{Introduction}

Dans un contexte de concurrence accrue, de recherche continue des pistes d'optimisation des coûts, et face à une demande de plus en plus exigeante en termes de qualité, de prix, de rapidité et d'innovation, les entreprises sont amenées à modifier en profondeur leurs modes d'organisation et de gestion. 
Les entreprises industrielles externalisent de plus en plus des activités jugées non stratégiques pour se concentrer sur leurs métiers de base. Le poids des achats ne cesse pas d'augmenter; ils représentent aujourd'hui entre 50 à $70 \%$ des coûts de production (Aguezzoul \& Ladet, 2009; Prajogo et al., 2012). Les fournisseurs jouent désormais des rôles stratégiques dans les organisations et permettent la création d'un avantage concurrentiel (Jabbour \& Jabbour, 2009).

Le recours à l'externalisation est économiquement très attractif. Les motifs les plus apparents qui poussent les entreprises à cette pratique sont tout d'abord un objectif de réduction des coûts et des délais, une recherche d'une flexibilité accrue ainsi que la valeur ajoutée apportée par ces fournisseurs experts dans leurs domaines. Toutefois, cette stratégie n'est pas sans risque pour le donneur d'ordres, surtout en termes de qualité et délai, dans la mesure où une grande partie de sa performance se trouve entre les mains des fournisseurs (Hohmann, 2004).

Aujourd'hui, praticiens comme chercheurs s'accordent à souligner le rôle crucial et critique des fournisseurs dans le sens où ils exercent une influence majeure dans le succès ou l'échec d'une entreprise (Colin \& Paché, 2000; Prajogo et al., 2012).

Les organisations sont confrontées alors aux problématiques relatives au management de ses différentes externalisations (Boissinot, 2008). La mission de gestion et de soutien de la performance des fournisseurs est devenue très critique dans la gestion des organisations et des chaînes d'approvisionnement (Schoenherr et al., 2012).

Cette étude, en sciences de gestion, s'inscrit dans le champ des recherches en Supply Chain Management «SCM». Elle s'intéresse principalement à la gestion de la partie amont de la chaine « Upstream Supply Chain » qui met en relation le donneur d'ordres «client» avec ses fournisseurs.

Plusieurs recherches dans la littérature ont traité la problématique de gestion des fournisseurs et son impact sur la performance de la relation. Certains chercheurs ont étudié l'impact direct de la gestion des fournisseurs sur la performance des entreprises, sans explorer cette performance comme une variable modératrice. (e.g. Flynn, Schroeder \& Sakakibara, 1995 ; Tan, Kannan, \& Handfield, 1998). Pour d'autres, ils ont mesuré l'effet des pratiques de gestion des fournisseurs sur la performance de ces derniers avant de traiter l'impact sur celle de l'entreprise cliente (Vonderembse \& Tracey, 1999; Park \& Hartley, 2002).

La gestion de la relation avec les fournisseurs comprend des activités telles que : 
- $\quad$ La sélection et le développement des fournisseurs (Vonderembse \& Tracey, 1999; Park \& Hartley, 2002; Hohmann, 2004; Park et al., 2010; Chen, 2011; Akamp \& Müller, 2011; Samson, 2013);

- $\quad$ Le développement des relations de partenariat à long terme avec les fournisseurs stratégiques (Hohmann, 2004; Prahinski et al., 2002; Li et al., 2004; Prajogo et al., 2011; Samson, 2013);

- $\quad$ L'intégration logistique (Prajogo et al., 2011; Akamp \& Müller, 2011; Samson, 2013);

- L'évaluation et le suivi de la performance des fournisseurs (Hohmann, 2004; Prajogot al., 2011; Akamp \& Müller, 2011; Samson, 2013);

- $\quad$ Le contrôle et l'audit des fournisseurs (Hohmann, 2004 ; Akamp \& Müller, 2011 ; Samson, 2013).

Dans cette étude, on essaie d'explorer les meilleures pratiques de gestion des fournisseurs utilisées pour assurer la performance des approvisionnements dans le secteur automobile au Maroc. Concrètement, l'objectif de cette recherche est de mettre le point sur l'état des lieux et les perspectives d'application d'une démarche de sécurisation et d'évaluation de la performance des fournisseurs, axée sur les pratiques de gestion et basée sur la combinaison des audits qualité et logistique.

La première partie de cet article est consacrée à la manière dont la littérature traite de la question. La deuxième partie présente la méthodologie employée. Dans la troisième et dernière partie, nous présentons l'interprétation de l'étude de cas réalisée auprès du constructeur automobile Renault Tanger au Maroc.

\section{Le pilotage de la performance des fournisseurs par les pratiques de SCM Concept de Supply Chain Management}

Le concept « Supply Chain Management » date du début des années 1980 et a pris toute son ampleur dans les années 1990 avec le besoin, pour les entreprises, de répondre rapidement aux demandes de plus en plus exigeantes des clients.

Plusieurs définitions ont été données à ce concept; nous retenons celles du Council of Supply-Chain Management Professionals (CSCMP) en 2005 : « Le management de la Supply-Chain comprend la planification et la gestion de toutes les activités relevant de la recherche des fournisseurs, de l'approvisionnement, de la transformation et toutes les activités relatives au management logistique. Cela inclut tout particulièrement la coordination et la coopération entre les partenaires du canal, qui peuvent être des fournisseurs, des intermédiaires, des prestataires de services et des clients. Le management de la Supply-Chain intègre donc le management de l'offre et celui de la demande, dans l'entreprise comme entre entreprises. Les fournisseurs constituent un maillon important de cette chaine logistique qui comporte une 
importance particulière. Ainsi, la gestion des fournisseurs préoccupe la plupart des entreprises commerciales et industrielles soucieuses d'améliorer leurs performances.

\section{Les relations client-fournisseurs}

La place des achats en tant que fonction stratégique au sein des entreprises a commencé à s'imposer dans les années 90 sous l'influence de deux facteurs : le recentrage des entreprises sur leur métier de base et la globalisation (Durand \& Faultrier, 2007).

Les achats occupent, aujourd'hui, une place importante dans la gestion de la plupart des entreprises vu les avantages qui peuvent être tirés d'une relation réussie avec les fournisseurs.

Les relations client-fournisseurs sont des processus d'interaction et d'échange entre une organisation cliente (acheteur ou consommateur) et une organisation fournisseur (vendeur ou producteur) (Guibert, 1996 ; Donada \& Nogatchewsky, 2005). Il s'agit d'une collaboration dans la durée et d'une mise en commun des compétences et des ressources pour concevoir et réaliser un produit ou processus (Donada, 1997).

\section{Les enjeux et les risques des relations client-fournisseur}

Dire que le risque est inhérent à la vie des entreprises et donc aux flux qui y circulent en interne comme aux interfaces est une évidence (Laville, 2006). En amont de la Supply Chain, les achats auprès des fournisseurs peuvent être la source d'une altération des capacités de production de l'entreprise, ainsi que de la non-satisfaction des clients, notamment du fait du non-respect des délais d'approvisionnement ou de la mauvaise qualité des produits (Jenn, 2008). Pour cette raison, certaines entreprises sont contraintes à tirer vers le haut les performances de certains de leurs fournisseurs stratégiques, afin qu'elles-mêmes puissent maintenir leurs résultats, voire tout simplement continuer à fonctionner (Laville, 2006). Les risques liés à la relation avec les fournisseurs font partie de la catégorie des risques opérationnels ou d'exploitation.

\section{La performance des fournisseurs}

La performance est un concept à la mode qui est largement utilisé dans le monde des affaires. En effet, la notion de performance est au cœur de toutes les démarches d'évaluation des entreprises et des organisations (Salgado, 2013).

La performance des fournisseurs, dans toutes ses dimensions (opérationnelle, financière, organisationnelle, etc.) impacte considérablement l'efficacité et l'efficience du client acheteur. La performance d'une relation client-fournisseur correspond au degré 
d'accomplissement des objectifs des partenaires de la relation (Anderson \& Coughlan, 2002).

L'évaluation de la performance est un facteur clé de motivation dans le monde des affaires avec un effet direct sur la performance. Autrement dit, « on n'obtient que ce qu'on mesure » (Cousins et al., 2008). Donc, le suivi et la gestion de la performance des fournisseurs est un levier important pour assurer l'efficacité et l'efficience de la chaine d'approvisionnement.

\section{La gestion des fournisseurs}

La gestion des fournisseurs en amont est un aspect clé dans le SCM, car cette relation met en jeu la performance de l'entreprise acheteuse et impacte significativement le coût, la qualité, le délai, la réactivité et la position concurrentielle des entreprises.

L'importance de la gestion des fournisseurs est largement reconnue dans la littérature sur le SCM. En effet, avec l'intensification de la concurrence à l'échelle internationale, la multiplication des opérations d'externalisation et l'augmentation du poids des achats dans la valeur des produits, les entreprises ont pris conscience du fait qu'il ne suffit plus de se concentrer sur l'amélioration des processus internes, mais il faut aussi veiller à assurer la performance le plus en amont possible dans les relations avec les fournisseurs.

La gestion des fournisseurs ne se limite pas à la gestion des transactions entre les parties et à la négociation des prix intéressants; elle s'intéresse à un éventail plus large des sujets relatifs à la relation. Aujourd'hui, l'objectif d'un gestionnaire des fournisseurs est d'assurer un flux optimal des matières achetées en termes de qualité, de prix, d'innovation, etc. (Szwejczewski et al., 2005).

\section{Les pratiques de la SCM liées à la gestion des fournisseurs}

Fondées sur le principe de la coordination entre les fonctions de l'entreprise et la coopération avec les partenaires, les pratiques de SCM ont connu un succès considérable dans l'amélioration de la performance des chaines logistiques. Néanmoins, malgré leur popularité dans le milieu académique et professionnel, il n'existe pas de véritable consensus sur la définition-même du concept ni sur les dimensions organisationnelles permettant sa mise en œuvre (Brulhart \& Moncef, 2009). De même, malgré le développement du concept de SCM, les études qui mettent en relation les pratiques de SCM (PSCM) et la performance des entreprises restent dispersées et incomplètes (Li et al., 2004 ; Chen \& Paulraje, 2004 ; Koh et al., 2007 ; Brulhart \& Moncef, 2009). Les pratiques de SCM sont définies comme un ensemble d'activités menées dans une organisation pour assurer une gestion efficace de son SC (Suhong Li et al., 2006). Il s'agit d'un ensemble de pratiques relatives à un mode de management dans une optique de SCM 
(Brulhart \& Moncef, 2009). D'après Li et al. (2005) et Koh et al. (2007), les pratiques de SCM se définissent comme les activités déployées dans une organisation afin de permettre la mise en œuvre réussie du SCM au sein d'un système de valeur.

La littérature est assez abondante en ce qui concerne l'identification des PSCM et l'étude de leur impact sur la performance de la SC. Certains chercheurs ont étudié les pratiques relatives à la gestion amont de la SC et le mode de gestion de la relation avec les fournisseurs (Narasimhan \& Jarayam, 1998 ; Shin et al., 2000 ; Pressuti, 2003 ; Chen \& Paulraj, 2004).

D'autres ont privilégié la gestion aval de la $\mathrm{SC}$ et se focalisent sur la gestion de la relation avec les clients (Tan, 2002 ; Min \& Mentzer, 2004 ; Moncef, 2008), alors que d'autres auteurs ont étudié les pratiques qui touchent la gestion globale de SC (Douglas M. Lambert et al., 2001 ; Rudberg \& Olhager, 2003 ; Nathalie Fabbe-Costes, 2005). Pour notre cas, nous nous focaliserons sur les pratiques de SCM liées à la gestion de la relation avec les fournisseurs.

Le tableau 1 reprend les principaux apports de la littérature sur les pratiques de SCM.

Tableau 1. Les principaux apports relatifs aux pratiques de SCM

\begin{tabular}{|c|c|}
\hline Auteurs & PSCM identifiées \\
\hline Donlon (1996) & $\begin{array}{c}\text { Relations de long terme avec les fournisseurs, réduction du } \\
\text { nombre de fournisseurs, qualité des fournisseurs, implication des } \\
\text { fournisseurs dans la conception et la production de l'offre. }\end{array}$ \\
\hline $\begin{array}{c}\text { Alvarado \& Kotzab } \\
\text { (2001) }\end{array}$ & $\begin{array}{c}\text { Focalisation sur les compétences centrales, systèmes de } \\
\text { coordination }\end{array}$ \\
inter-organisationnelle (EDI).
\end{tabular}




\begin{tabular}{|c|c|}
\hline Chow et al. (2008) & Partenariat fournisseurs, partage d'informations. \\
\hline Hsu et al (2009) & $\begin{array}{c}\text { Améliorer la capacité des fournisseurs dans le « Just-in-time », La } \\
\text { proximité géographique des fournisseurs, Partage des } \\
\text { informations, Améliorer l'intégration des activités, Communiquer } \\
\text { les futurs besoins stratégiques, suivre la performance des } \\
\text { fournisseurs }\end{array}$ \\
\hline $\begin{array}{l}\text { Brulhart \& Moncef } \\
\text { (2009) }\end{array}$ & $\begin{array}{l}\text { L'échange et le partage d'informations, la gestion partenariale de } \\
\text { la relation fournisseur }\end{array}$ \\
\hline $\begin{array}{l}\text { Vanichchinchai \& } \\
\text { Igel (2011) }\end{array}$ & Gestion des informations, gestion du partenariat, \\
\hline Silva et al (2012) & $\begin{array}{l}\text { Développement des fournisseurs, suivi de la performance des } \\
\text { fournisseurs, intégration des fournisseurs, les technologies } \\
\text { d'information et de communication }\end{array}$ \\
\hline $\begin{array}{l}\text { Karimiet Rafiee } \\
(2014)\end{array}$ & $\begin{array}{l}\text { Partenariat stratégique avec les fournisseurs, niveau et qualité de } \\
\text { partage des informations }\end{array}$ \\
\hline Sharma et al. (2008) & $\begin{array}{l}\text { Implication des fournisseurs, simplification et informatisation du } \\
\text { processus d'achat, gestion de la performance des fournisseurs, } \\
\text { partenariat stratégique avec les fournisseurs, proximité des } \\
\text { fournisseurs, équipe transverse et multifonctionnelle, intégration } \\
\text { des processus, travail avec les fournisseurs sur la qualité, audit } \\
\text { qualité, implication des fournisseurs, formation, partage des } \\
\text { informations }\end{array}$ \\
\hline
\end{tabular}

Source : élaboration personnelle

\section{L'audit combiné qualité et logistique}

La convergence et la synergie des approches «Qualité » \& « Logistique »

Comme les marchés mondiaux évoluent, les gestionnaires de la chaîne d'approvisionnement sont confrontés à de nombreux nouveaux défis, car les approches classiques de gestion se révèlent de plus en plus inefficaces. Ainsi, une approche globale de gestion de la chaine de valeur s'impose ; elle vise à la livraison des produits attendus dans les quantités désirées, au niveau de qualité requis, au bon endroit, en temps en l'heure, en respectant les exigences et/ou engagements de service et au moindre coût.

Selon Hohmann (2004), « Les services chargés des questions qualité et logistique ont longtemps vécu côte à côte, sans trop se fréquenter. Leur rapprochement est inévitable, car ces deux fonctions désormais transverses partagent certaines caractéristiques et un certain nombre d'objectifs ». Les fonctions «qualité et logistique » ont évolué et passé par la même trajectoire : du niveau opérationnel au niveau stratégique. La synergie entre les deux dimensions «qualité et logistique » a été illustrée par le modèle du cône de sable de Ferdows et De Meyers (1990) (Figure 1). Selon cette théorie, l'excellence industrielle est probablement basée sur un ensemble de principes de base qui sont plus faciles à mettre en place en respectant une séquence où 
chaque effort entrepris peut servir de tremplin aux actions à venir et que les effets en sont plus cumulatifs qu'exclusifs (Jobin \& Friel, 2001).

Figure 1. Le modèle du cône de sable

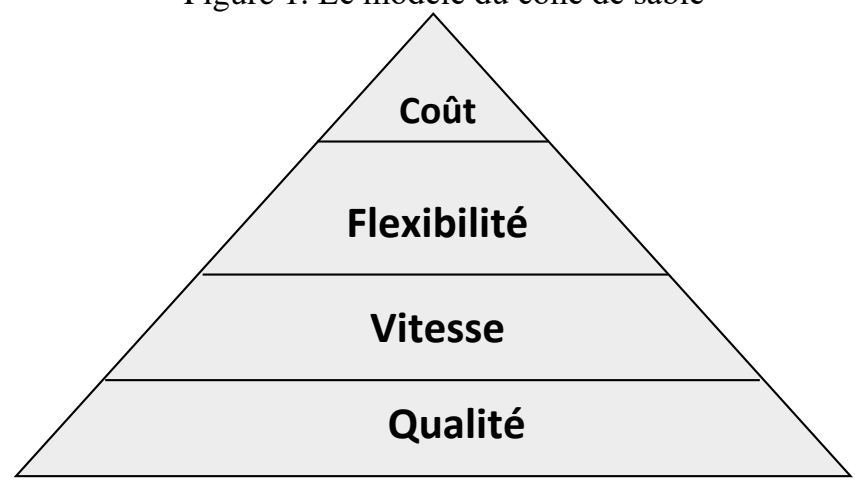

Source : Ferdows and De Meyer (1990), cité par JOBIN et FRIEL, (2001)

Selon Ferdows et De Meyer (1990), on peut difficilement aspirer à une meilleure performance en termes de coûts si, préalablement, des gains en matière de qualité, de fiabilité et de vitesse n'ont pas été réalisés.

La pierre d'angle de leur article était que, contrairement à la vision traditionnelle qui considère le coût, la qualité, la fiabilité et la flexibilité comme des objectifs divergents qui doivent faire l'objet d'un arbitrage douloureux, ces capacités concurrentielles seraient en fait additives et hiérarchisées. La création de valeur dans ce contexte serait le fruit d'un travail hiérarchisé d'amélioration, débutant par l'amélioration de la qualité des produits suivi de l'amélioration de la fiabilité et de la rapidité du système pour finalement réaliser des gains en matière de réduction des coûts.

Aujourd'hui, la poursuite simultanée des objectifs «qualité et logistique » est efficace en raison de la synergie créée entre les deux dimensions. Par exemple, les pratiques de gestion de la qualité sont utiles non seulement pour atteindre les objectifs de qualité, mais aussi dans la réalisation d'autres objectifs stratégiques notamment la performance logistique. La satisfaction des clients constitue le point commun et l'objectif ultime des deux démarches "qualité et logistique ». Flynn et Flynn (2005) ont prouvé la nécessité d'intégrer la gestion de la qualité et la gestion de la Supply Chain pour réaliser des bonnes performances.

\section{Principe et origine de l'audit combiné « qualité et logistique »}

Selon Christian HOHMANN, l'approche d'audit combiné est fondée sur le constat que les objectifs et les missions des fonctions qualité et logistique convergent naturellement. Il suffit donc d'outiller cette démarche, en combinant opportunément ce que chacune des fonctions avait déjà développé et en l'alignant sur les objectifs de sécurisation ». (Hohmann, 2004). 
L'audit qualité est un outil indispensable pour faire vivre la qualité au sein de l'entreprise. En interne, il est source de progrès et garant de l'efficacité du système qualité. En externe, il permet d'évaluer, de sélectionner les fournisseurs et de créer avec eux une relation durable et efficace.

La norme ISO 10011-1 remplacée par la norme 19011 : 2011 définit l'audit logistique comme " un examen méthodique et indépendant, en vue de déterminer si les activités et résultats relatifs à la logistique satisfont aux dispositions prétablies (cahiers des charges, normes...), si ces dispositions sont mises en œuvre de façon efficace et si elles sont aptes à atteindre les objectifs.

Selon le guide EA (European Co-operation for Accreditation) pour l'application de la norme ISO/CEI 17021: 2006 pour les audits combinés, « Un audit combiné est un audit du (des) système(s) de management d'une organisation portant sur un ensemble de deux critères d'audits/normes au minimum réalisé au même moment ». L'idée principale des deux audits «qualité et logistique » est que :

- $\quad$ D'une part, l'audit logistique désigne à partir de son appellation le respect de tous les composants de la chaine logistique et de mesurer la performance de la chaine.

- D'autre part, l'audit qualité sert à respecter les conditions auprès de la clientèle d'une manière à fidéliser les clients et les satisfaire et d'autres manières à garder l'image fidèle de l'entreprise productive.

Partant de ce constat, l'audit qualité a pour objectif de vérifier entre deux intervalles planifiés, si l'ensemble des points audités se sont dégradés ou non ou si le plan d'amélioration continue de la qualité a apporté une meilleure maîtrise de la lutte contre la non-qualité. Toutefois, le respect des normes de la qualité à tel point ne satisfera pas le client à un large niveau car, d'un point de vue clientèle, la notion de coût et du délai de livraison et de la disponibilité du produit reste pour lui des points d'équilibre entre ce qui est estimé et prévu et ce qui est adapté sur terrain.

Déduisant donc que l'audit qualité peut assurer l'amélioration de la qualité, il ne pourra pas assurer tout seul l'équilibre du triptyque coût, qualité, délai. La combinaison entre les deux audits peut avoir un effet positif sur la continuité de la production et de l'amélioration relation client/fournisseurs en respectant le fil de satisfaction des trois notions coût, qualité et délai et en réalisant une progression de la performance de l'entreprise.

La combinaison des deux audits se base essentiellement sur l'audit du système de management d'une organisation partant sur un ensemble de deux critères d'audits/normes au minimum réalisé au même moment. Cette combinaison n'est autre chose que la combinaison entre deux ou plusieures normes d'audits effectués en même temps. La construction de la combinaison d'audit peut se retrouver dans différents niveaux d'intégration. 
Comme les performances qualité ont également un impact sur les performances logistiques et inversement, (deux audits distincts qualité et logistique se recouvrent partiellement), il est intéressant de combiner les deux approches en une seule. Cela est plus facilement acceptable par les fournisseurs et plus économique pour le client qui envoie ses experts.

De plus, en envoyant une équipe mixte, le donneur d'ordre accélère les transferts de savoir entre qualiticiens et logisticiens, leur donnant ainsi une double compétence dans des domaines qui convergent naturellement. Progressivement, en libérant des experts multi compétents, le client pourra multiplier les audits de ce type pour mieux contrôler l'amont de sa propre supply chain. Mieux encore, il aura la possibilité de former des spécialistes chez ses fournisseurs pour qu'ils transmettent ces dispositifs à leurs propres fournisseurs et diffusent ainsi nons seulement le contrôle, mais aussi les compétences et les bonnes pratiques dans le réseau des fournisseurs.

\section{Question de recherche et modèle conceptuel}

La revue de la littérature liée au sujet nous a permis de comprendre que le pilotage de la performance des fournisseurs nécessite la mise en place d'une démarche stratégique de sécurisation des approvisionnements en matière du triptyque "qualité, coût et délai », basée sur un ensemble de pratiques de gestion. L'audit combiné « qualité et logistique » est une pratique émergente qui se rajoute aux autres pratiques classiques pour renforcer le système de sécurisation.

A travers cette étude, nous essayons de comprendre comment un système de pilotage de la performance des fournisseurs est mis en place et quel est le rôle de chaque acteur? Nous essayerons de répondre à la question de recherche suivante :

Comment un constructeur automobile arrive-t-il à piloter la performance et assurer la sécurisation de sa relation avec les fournisseurs équipementiers par les pratiques de Supply Chain Management et quel est l'apport de l'audit combiné « qualité et logistique»?

En effet, l'analyse des relations entre les variables mentionnées sur la figure $\mathbf{n}^{\circ} \mathbf{2}$ nous permettra de mieux comprendre notre problématique. 
Figure 2. Les concepts de la recherche

\section{Performance des fournisseurs}

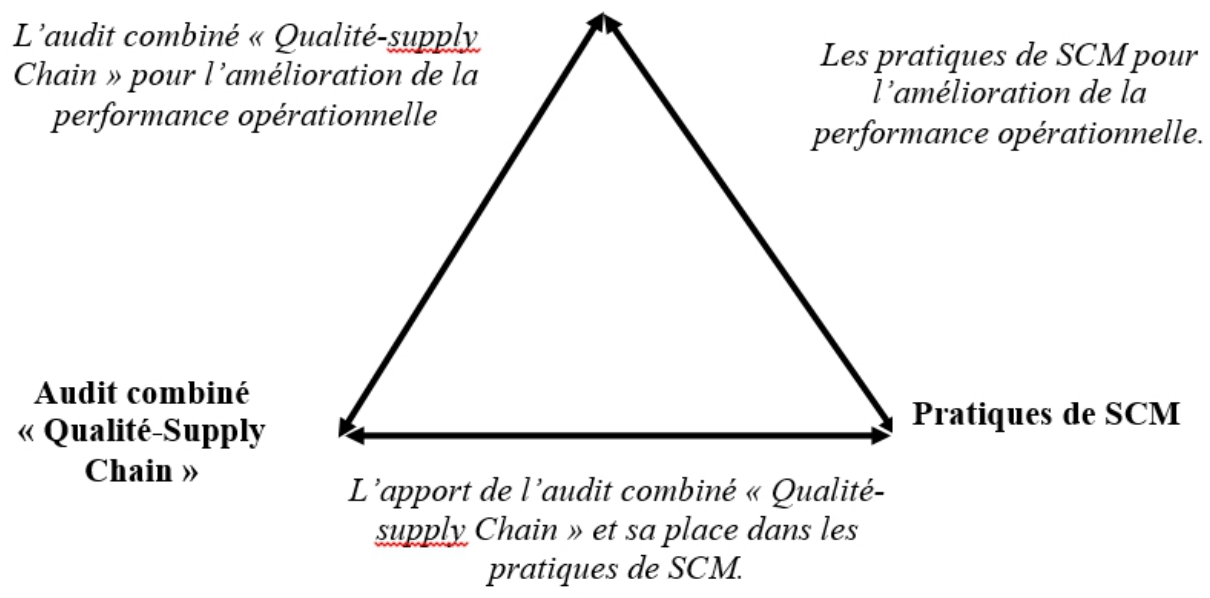

Source : élaboration personnelle

Nous résumons notre modèle par le schéma suivant (Figure 3). Nous intégrons en même temps les six variables :

Figure 3. Modèle conceptuel d'un système de pilotage de la performance des fournisseurs

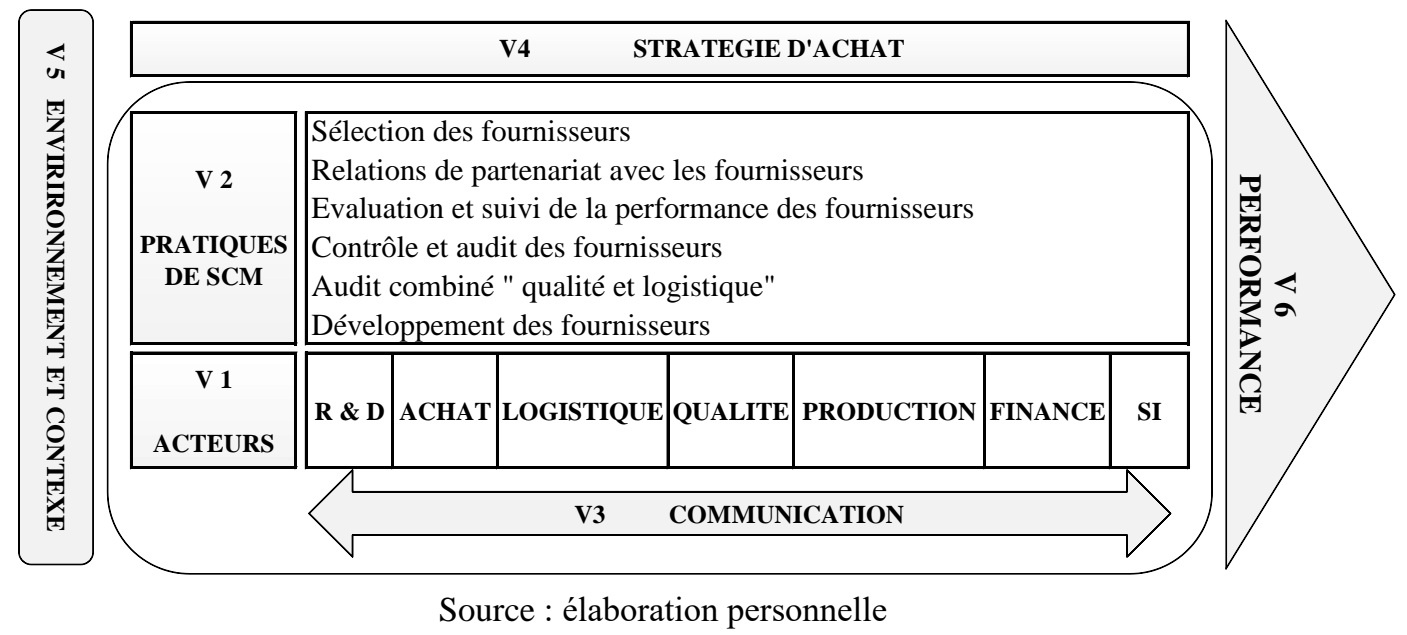

Notre modèle se situe à un double niveau d'analyse : organisationnel et processuel. Au niveau organisationnel, il permet d'expliquer la structure du système de pilotage de la performance des fournisseurs, ainsi que les éléments composants. Au niveau processuel, il permet d'expliquer les pratiques de gestion qui assurent la réussite de la relation avec les fournisseurs. Le modèle permet également d'établir le lien entre ces deux niveaux. Le pilotage de la 
performance des fournisseurs nécessite la participation de tous les acteurs, ces derniers liés par des canaux de communication.

Le choix des variables a été effectué suite à la revue de littérature sur les différents modèles qui ont traité la problématique de la gestion des fournisseurs.

Tableau 2. Description des variables du modèle de recherche

\begin{tabular}{|c|c|}
\hline $\begin{array}{l}\text { Variable } 1: \\
\text { Les acteurs }\end{array}$ & $\begin{array}{l}\text { Les pratiques de SCM sont conçues et mises en œuvre } \\
\text { par des acteurs généralement issus de différentes } \\
\text { fonctions et ayant des profils différents. }\end{array}$ \\
\hline $\begin{array}{c}\text { Variable 2: } \\
\text { Les pratiques de SCM }\end{array}$ & $\begin{array}{l}\text { Nous avons sélectionné les pratiques de SCM liées à } \\
\text { notre sujet de recherche qui est la gestion des } \\
\text { fournisseurs. Nous avons pris en compte la différente } \\
\text { phase de la relation avec les fournisseurs, depuis la } \\
\text { sélection jusqu'au développement. }\end{array}$ \\
\hline $\begin{array}{c}\text { Variable } 3: \\
\text { Les canaux de communication }\end{array}$ & $\begin{array}{c}\text { La communication est un maillon très important dans le } \\
\text { processus de pilotage de la performance, que ce soit } \\
\text { interne ou externe. }\end{array}$ \\
\hline $\begin{array}{c}\text { Variable 4: } \\
\text { La stratégie d'achat }\end{array}$ & $\begin{array}{l}\text { La stratégie d'achat conditionne les types des pratiques } \\
\text { à mettre en œuvre et le profil des acteurs intervenants. }\end{array}$ \\
\hline $\begin{array}{c}\text { Variable } 5: \\
\text { Environnement et contexte }\end{array}$ & $\begin{array}{l}\text { L'environnement est une variable explicative de } \\
\text { plusieurs variables. }\end{array}$ \\
\hline $\begin{array}{l}\text { Variable 6: } \\
\text { Performance }\end{array}$ & Il s'agit principalement d'une variable à expliquer. \\
\hline
\end{tabular}

Source : élaboration personnelle

\section{Méthode de recherche}

\section{Présentation de l'étude de cas}

Notre recherche se conforme à analyser le système de pilotage de la performance des fournisseurs, ses pratiques, ses mécanismes et ses acteurs. Nous visons également à comprendre l'état des lieux et les perspectives d'application de l'audit combiné « qualité et logistique ». Dans notre modèle conceptuel, nous proposons un système de pilotage et ses principaux éléments composants. Ainsi, compte tenu de la nature et de l'objet de notre recherche, et des multiples unités à analyser, nous avons mis en place un dispositif empirique basé sur l'approche qualitative, et en particulier, sur la méthode de cas.

Nous avons choisi le constructeur automobile «Renault Tanger Exploitation », avec ses différentes fonctions intervenantes dans le processus de pilotage de la performance des fournisseurs comme unités d'analyse.

Selon (Yin, 1984), une étude de cas est « une enquête empirique qui examine un phénomène contemporain au sein de son contexte réel lorsque les 
frontières entre phénomène et contexte ne sont pas clairement évidentes et pour laquelle de multiples sources de données sont utilisées » (Yin, 1984, p. 17).

L'étude de cas est considérée comme une étude de sites au sens d'Albarello (2003) : « Une étude de sites étudie des phénomènes dans un petit groupe ou dans un service, un département, une organisation ou une communauté ». La recherche in situ apparaît par ailleurs la plus appropriée si l'objectif de la recherche est de décrire des « pratiques de gestion » au sens de Dumez (1988).

Le choix de l'étude de cas comme stratégie de recherche se justifie par le caractère exploratoire de notre recherche qui aspire à étudier un phénomène contemporain dans le contexte de la vie réelle (Benbasat et al., 1987 ; Yin, 1984). Ce choix a été motivé par notre objectif de procéder à une analyse plus fine d'une société qui a mis en place des pratiques de SCM, plutôt que d'établir des comparaisons inter-sociétés.

Notre stratégie de recherche s'appuie sur l'étude de cas unique, le cas de Renault Tanger, et nous considérerons plusieurs unités d'analyse. Le recours à une étude de cas unique est préconisé dans trois situations spécifiques (Yin, 1990) : «pour tester une théorie (avec l'objectif de la confirmer, de la réfuter ou de la compléter), pour révéler un phénomène non rare mais auquel la communauté scientifique a difficilement accès ou encore si le cas étudié est rare ou unique ». Dans notre cas, il s'agit d'un phénomène non rare mais sur lequel on a encore peu de connaissance.

Notre analyse porte sur une pratique managériale peu étudiée dans la littérature «Le pilotage de la performance des fournisseurs par les pratiques de Supply Chain Mangement et l'apport de l'audit combiné «qualité et logistique ».

Cet objet d'étude devient nécessaire à étudier avec précision. L'étude de cas n'est donc pas un choix méthodologique mais le choix d'un objet à étudier (Stake, 1994).

Le pilotage de la performance des fournisseurs par les pratiques de gestion, et en particulier par l'audit combiné « qualité et logistique », est un phénomène complexe qui devra être analysé en profondeur, et la présence du chercheur dans l'entreprise pendant une longue période parait comme le moyen privilégié pour étudier ce processus. Nous avons choisi donc la méthode de l'étude de cas par immersion en entreprise.

Le choix du secteur d'activité n'a pas été réalisé aléatoirement. En effet, dans le secteur automobile, les achats et les relations avec les fournisseurs sont considérés comme un enjeu stratégique, vu la valeur et le nombre important des pièces achetées qui rentrent dans la composition du produit final. En plus, les mécanismes et les pratiques de pilotage des fournisseurs sont plus avancés. Plusieurs chercheurs ont réalisé leurs travaux 
de recherche dans le secteur automobile (Donada, 1998 ; Dyer \& Ouchi, 1993 ; Dyer \& Chu, 2000 ; Anderson et al., 2000 ; Donada \& Garrette, 2001 ; Kotabe et al., 2003 ; Nogatchewsky, 2004).

Dans le contexte marocain, le secteur automobile constitue un véritable métier mondial du Maroc, surtout avec l'implantation des grands constructeurs mondiaux (Renault et PSA). Ces constructeurs ont affiché des objectifs ambitieux en termes d'une politique d'intégration locale qui vise à augmenter le taux des composants approvisionnés localement.

Ainsi, à travers cette recherche, nous souhaiterons mettre à la disposition des équipementiers et des fournisseurs marocains qui souhaitent améliorer leurs performances, un guide afin de décrocher des contrats à long terme avec les constructeurs automobiles.

Nous avons choisi de réaliser notre recherche du côté d'un constructeur automobile car ce dernier, par référence à la chaine de valeur automobile, est le premier responsable de la performance du produit final en termes de qualité, de coût et de délai.

La méthodologie adoptée est de type qualitatif, et nous optons pour une étude de cas in situ dans la société Renault Tanger Exploitation.

\section{Recueil et construction des données}

Pour notre cas, nous avons combiné trois types de méthodes pour la collecte des données : l'intervention et l'observation directe, l'entretien et la documentation.

Par rapport à notre statut et celui du participant qui observe, en tant que contrôleur de gestion au sein de la société et en plus de mon travail quotidien d'analyse et de Reporting, j'ai assisté à ses réunions avec les différentes entités impliquées dans la relation avec les fournisseurs (Achats, ingénierie, qualité, logistique, Système d'information, fabrication, etc.). Nous avons présenté notre statut d'observateur en exposant notre objectif, notre forme d'investigation, tout en gardant notre neutralité.

Dans notre étude de cas, nous avons mené une série des entretiens individuels en face à face. Pour pouvoir collecter des données de qualité, nous avons préparé en avance le format de l'entretien. L'objectif était de cadrer au maximum l'entretien, sans pour autant contraindre le répondant. Pour cela, nous avons conçu des guides d'entretien avec de questions générales pour laisser une certaine ouverture à l'interviewé et d'accroître sa liberté de réponse.

Le guide était composé de différents axes modulables en accord avec la fonction de l'acteur. Nous avons opté donc pour des entretiens de type semi directif pour laisser une certaine liberté au répondant.

Nous avons réalisé des entretiens auprès des acteurs impliqués dans le processus de pilotage de la performance des fournisseurs. Les acteurs 
interrogés sont situés dans les différents niveaux hiérarchiques de la société. Nous les avons regroupés en trois niveaux organisationnels : corporatif, tactique et opérationnel. (Tableau 3)

Tableau 3. Catégorie et niveau hiérarchique des acteurs interrogés

\begin{tabular}{|c|c|c|}
\hline $\begin{array}{c}\text { Niveau } \\
\text { hiérarchique }\end{array}$ & Description & Entretiens réalisés \\
\hline $\begin{array}{l}\text { Niveau } \\
\text { Corporatif }\end{array}$ & $\begin{array}{l}\text { Le directeur de l'usine et les } \\
\text { autres membres du comité de } \\
\text { direction ; ce sont les acteurs } \\
\text { qui participent à la prise des } \\
\text { décisions et qui supervisent le } \\
\text { processus de pilotage de la } \\
\text { performance des fournisseurs. }\end{array}$ & $\begin{array}{c}\text { Directeur de l'usine } \\
\text { Directeur Logistique Industrielle } \\
\text { Usine } \\
\text { Directeur Qualité Usine } \\
\text { Directeur de Fabrication } \\
\text { Directeur Technique } \\
\text { Directeur Finance et contrôle de } \\
\text { gestion } \\
\text { Chef de Département Ingénierie } \\
\text { Maroc } \\
\text { Chefs de département fabrication }\end{array}$ \\
\hline $\begin{array}{l}\text { Niveau } \\
\text { Tactique }\end{array}$ & $\begin{array}{l}\text { Les pilotes du progrès des } \\
\text { fournisseurs et les pilotes des } \\
\text { systèmes de management } \\
\text { qualité et logistique. Ils ont } \\
\text { pour mission d'assurer le } \\
\text { pilotage de la performance du } \\
\text { fournisseur conformément aux } \\
\text { exigences du niveau } \\
\text { Corporatif tout en assurant les } \\
\text { rôles d'information, de } \\
\text { coordination et de relais avec } \\
\text { le niveau opérationnel. }\end{array}$ & $\begin{array}{c}\text { Pilotes Performance Logistique } \\
\text { Fournisseurs } \\
\text { Pilote du système de management } \\
\text { qualité } \\
\text { Pilotes Progrès Fournisseur } \\
\text { Contrôle de gestion Achats, } \\
\text { logistique et ingénierie } \\
\text { Auditeurs qualité } \\
\text { Consultants Qualité Fournisseur } \\
\text { Consultant Développement } \\
\text { Fournisseur } \\
\text { Responsable Performance et Qualité } \\
\text { Manager Performance Fournisseur } \\
\text { Local }\end{array}$ \\
\hline $\begin{array}{c}\text { Niveau } \\
\text { Opérationnel }\end{array}$ & $\begin{array}{l}\text { Les acteurs concernés } \\
\text { directement par la } \\
\text { performance des fournisseurs, } \\
\text { en particulier les personnes de } \\
\text { la fabrication, la qualité et la } \\
\text { logistique, etc. }\end{array}$ & $\begin{array}{c}\text { Acheteurs } \\
\text { Qualiticien Achats } \\
\text { Chefs d'atelier } \\
\text { Technicien Qualité Fournisseur }\end{array}$ \\
\hline
\end{tabular}

Source : élaboration personnelle

Les documents que nous avons utilisés et analysés concernent essentiellement la stratégie d'achat, les pratiques de SCM, l'audit fournisseur, la politique qualité, l'audit fournisseur, etc. Il s'agit essentiellement des procédures, des brochures, des notes, des rapports d'audit, des guides destinés aux fournisseurs, des comptes rendus de réunions, des mémoires universitaires disponibles sur internet, etc. L'accès à l'intranet de la société a été d'une grande utilité pour nous. Nous avons aussi utilisé une documentation externe sous forme d'articles et publications dans des sites spécialisés. 
L'analyse documentaire a été exploitée pour confirmer et valider les données recueillies durant les observations et les entretiens.

\section{L'analyse de données}

Les analyses menées pendant le recueil des donnes ont consisté à condenser, réduire et à classifier les données à travers des transcriptions et des comptes rendus des entretiens dans des fiches d'analyse par entretien, des fiches de synthèse des observations et des fiches de synthèse des documents.

Après la phase de recueil des données, l'analyse des données a été menée sur deux niveaux :

- Premier niveau: la restitution des dimensions du processus de pilotage de la performance des fournisseurs à travers les discours des interviewés et des pratiques observées.

- Le deuxième niveau tente de construire une structure explicative enracinée dans les faits pour analyser les liens entre les différentes variables du processus de pilotage de la performance des fournisseurs par les pratiques de SCM. Ces deux niveaux d'analyse ont été assistés par les analyses préalables réalisées dans la phase de recueil des données. Ils se sont appuyés sur deux outils essentiels : le dictionnaire des thèmes empiriques et le dictionnaire des thèmes théoriques.

Pour le traitement et l'analyse des données, nous traitons les données recueillies manuellement sans faire appel à un logiciel d'analyse.

Durant cette recherche, on s'est efforcé de satisfaire aux critères de la validité et de la fiabilité de la recherche qualitative tels qu'ils sont définis par Mucchielli : la complétude, la saturation, l'acceptation interne, la cohérence interne, et la confirmation externe (Gambault, 2000, cité par Hattabou, 2011).

\section{Terrain de recherche}

On a choisi comme terrain de recherche le secteur automobile au Maroc à travers le constructeur automobile Renault Tanger. L'industrie automobile est l'un des secteurs industriels les plus étudiés par les chercheurs en gestion. Deux grandes raisons expliquent cette situation : tout d'abord, cette industrie présente des enjeux économiques considérables pour les pays producteurs; ensuite, c'est un immense champ d'innovation en matière d'organisation, tant au niveau de la production que de la conception (Donada, 2000).

La filière automobile fait intervenir de nombreux acteurs tout au long de la chaîne de valeur. Dans la partie amont, on classe couramment les fournisseurs du constructeur automobile (encore appelé « intégrateur » ou « $\mathrm{OEM} »$ pour « Original Equipement Manufacturer ») en rangs « 1 » à «n $»$ où le rang 1 est directement fournisseur du constructeur. 
L'échelonnement dépasse rarement 4, ce dernier étant typiquement un fournisseur de matières premières, alors que le rang 1 est presque toujours impliqué dans une relation de co-développement avec son client autour d'une pièce, d'un module ou d'un système (Souilah, 2009).

Dans cette recherche, on traite la relation du constructeur automobile avec ses équipementiers ou fournisseurs de $1^{\mathrm{er}}$ rang. Au Maroc, le seul constructeur automobile opérationnel aujourd'hui est Renault, avec ses deux usines: SOMACA (Société Marocaine de construction automobile) à Casablanca et Renault Tanger. Depuis son installation au Maroc, le groupe RENAULT n'a cessé de faire déployer la politique de l'intégration locale qui vise à augmenter le taux des composants approvisionnés localement. Cette tendance est motivée par les gains à réaliser en termes de proximité géographique, de baisse des coûts logistiques et risques de change. Toutefois, ces gains sont conditionnés par l'obtention des composants aux standards RENAULT, dans les conditions de coût, délais et qualités déterminées. (Ibn el Farouk et al.2009). « Il faudra parvenir à $60 \%$ de valeur locale pour l'usine de Tanger contre un peu plus de $40 \%$ aujourd'hui. C'est notre but. » -déclare Jacques Prost, Directeur Général de Renault Maroc.

\section{Interprétation des résultats}

La question fondamentale à laquelle nous souhaitions avoir une réponse est la suivante: comment relier les trois concepts «Pratiques de SCM », «Audit combiné : Qualité et Logistique» et «Performance des fournisseurs »?, cela en étudiant leurs interactions et les différents liens entre eux. Notre revue de littérature liée au sujet nous permet de répondre que les liens entre ces trois termes sont très étroits.

Cependant, cela s'applique-t-il au cas de Renault Tanger dans sa relation avec les fournisseurs équipementiers au regard de notre investigation théorique?

Nos analyses empiriques montrent que ces trois liens existent au sein de Renault Tanger parce que, tout d'abord, le pratiques de SCM relatives à la gestion des fournisseurs couvrent toutes les étapes de contrôle préconisées par Bouquin (2001) : la finalisation (avant l'action), le pilotage (pendant l'action) et la post-évaluation (après l'action). Nous avons constaté quelques aspects de l'audit combiné "qualité et logistique» dans certaines pratiques, notamment : la couverture simultanée des deux axes de performance «Qualité et logistique ", et l'implication d'une équipe multidisciplinaire (Acheteurs, qualiticiens, logisticiens, Ingénieurs, etc.). Ensuite, l'évaluation de la performance est présente dans la plupart des pratiques de SCM, à travers des indicateurs de suivi QCD.

La crise, qui n'a pas épargné les fournisseurs auprès de qui Renault achète les pièces attendues, a obligé Renault à s'adapter. Cette dernière a placé 
la sécurisation des approvisionnements et l'amélioration de la compétitivité parmi ses axes stratégiques. Cette compétitivité ne peut être atteinte qu'en établissant des relations de partenariat avec les fournisseurs.

Les interactions entres les variables analysées peuvent être schématisées comme suit :

Figure 4. Les interactions entre les variables

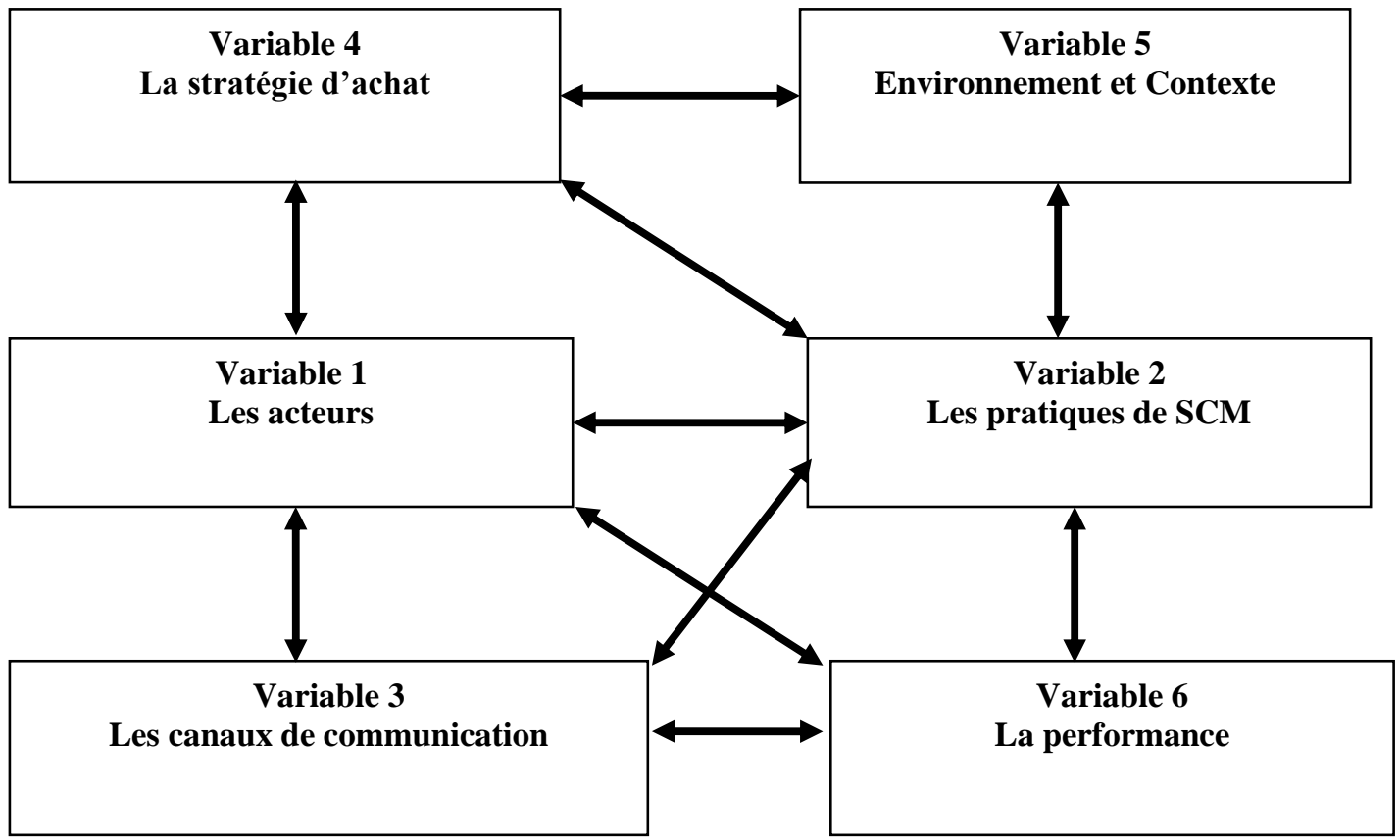

Source : élaboration personnelles

\section{La relation entre la variable 1 « les acteurs » et les autres variables}

- $\quad$ La diversité des métiers et des fonctions impliquées favorise la mise en place et l'exécution d'une panoplie des pratiques d'évaluation et de développement des fournisseurs ;

- La collaboration et l'implication des différents acteurs est une condition principale pour faire aboutir les pratiques de SCM et réaliser une bonne performance en termes de QCDDM (en anglais Quality, Cost, Delivery, Development, and Management);

- A travers la communication, que ce soit interne (réunions, mailing, tél, etc.) ou externe (EDI, journées fournisseurs, etc.), les acteurs participent à l'émergence d'une culture de performance et d'excellence dans la relation client-fournisseur. 


\section{La relation entre la variable 2 «les pratiques de SCM » et les autres variables}

- $\quad$ La nature et l'aspect du management touchés par les pratiques de SCM déterminent le profil des acteurs intervenants ;

- $\quad$ Le niveau de performance réalisé par le site est le résultat direct des pratiques de SCM durant toutes les phases de la relation avec les fournisseurs ;

- La pratique de l'audit combiné « qualité et logistique » en tant qu'un audit d'efficacité permet de réaliser les performances souhaitées, en tirant profit de la synergie entre la qualité et la logistique ;

- $\quad$ L'audit combiné « qualité et logistique » ne se substitue pas aux audits classiques de conformité ;

- $\quad$ La mise en place des pratiques de SCM nécessite un effort important en termes de communication (diffusion des résultats, sensibilisation, alerte, communication des objectifs, informations, etc.).

\section{La relation entre la variable 3 « les canaux de communication » et les autres variables}

- Le processus de communication traverse les différentes fonctions de l'entreprise. Cela nécessite un partage permanent des informations entre les différents acteurs ;

- La documentation et la formation sont indispensables pour la bonne exécution des pratiques de gestion des fournisseurs ;

- Une bonne communication favorise une bonne relation avec les fournisseurs pour arriver à une bonne performance.

\section{La relation entre la variable 4 « La stratégie d'achat » et les autres variables}

- $\quad$ La stratégie d'achat et les orientations de l'entreprise conditionnent la nature des pratiques de gestion des fournisseurs à mettre en place ;

- $\quad$ La stratégie d'achat pourra impacter les profils des acteurs à engager dans ce processus de pilotage ;

- La stratégie d'achat pourra convertir les contraintes de l'environnement en opportunités.

\section{La relation entre la variable 5 « Environnement et Contexte » et les autres variables}

- $\quad$ Le contexte et l'environnement de l'entreprise ont un impact direct ou indirect sur toutes les variables ;

- L'environnement et le contexte impactent le choix de la stratégie d'achat de l'entreprise ;

- L'environnement et le contexte impactent le choix de la nature des pratiques de SCM à mettre en place. 


\section{La relation entre la variable 6 « Performance » et les autres variables}

- Le niveau de performance réalisé conditionne le choix des pratiques de pilotage à mettre en place ;

- $\quad$ La performance réalisée impacte le profil et le niveau d'implication des acteurs ;

- $\quad$ Le niveau de performance atteint conditionne le choix de la fréquence, du canal et de la nature de l'information à communiquer.

L'analyse des résultats montre que le processus de pilotage de la performance des fournisseurs est un projet de toute l'entreprise qui implique plusieurs fonctions, d'une façon séparée ou dans le cadre des équipes transversales. Plusieurs pratiques de gestion des fournisseurs sont mises en place par Renault Tanger, par des acteurs de différents profils et durant toutes les phases de la relation avec les fournisseurs. La majorité des acteurs interviewés sont satisfaits des résultats des pratiques actuelles dont l'audit combiné « qualité et logistique », pratique qui a été appréciée par la plupart des acteurs comme une démarche innovante et mieux acceptable.

Ainsi, dans l'analyse des résultats, nous avons tiré une panoplie des bonnes pratiques qui peuvent être d'une grande utilité pour les managers.

Tableau 4. Bonnes pratiques managériales

\begin{tabular}{|c|c|}
\hline Leviers d'amélioration & Bonnes pratiques \\
\hline Les acteurs & $\begin{array}{c}\text { - Impliquer plusieurs métiers et fonctions dans le processus de } \\
\text { pilotage de la performance des fournisseurs (la gestion des } \\
\text { fournisseurs n'est pas l'apanage des achats); } \\
\text { - Former des équipes transverses composées de différents } \\
\text { profils afin s'assurer une couverture exhaustive des points liés à } \\
\text { la relation avec les fournisseurs ; } \\
\text { - Assurer une formation continue des différentes acteurs. }\end{array}$ \\
\hline $\begin{array}{c}\text { - Prévoir des pratiques qui couvrent les différentes phases de la } \\
\text { relation avec les fournisseurs : développement, vie série ; } \\
\text { - Prévoir des pratiques spécifiques à un certain nombre de } \\
\text { situations : nouveau fournisseur, nouveau site, importance du } \\
\text { produit livré, défaillance du fournisseur sur un axe de } \\
\text { performance, etc ; } \\
\text { - Déployer les mêmes types de pilotage par les fournisseurs de } \\
\text { rang 1 auprès de leurs fournisseurs de rang n ; } \\
\text { - En fonction de l'évolution de la relation et de la maturité des } \\
\text { fournisseurs, il faut veiller à supprimer les contrôles et les } \\
\text { pratiques supplémentaires et inutiles ; } \\
\text { - La pratique de l'audit combiné « qualité et logistique » en tant } \\
\text { qu'un audit d'efficacité permet de réaliser les performances } \\
\text { souhaitées, en tirant profit de la synergie entre la qualité et la } \\
\text { logistique ; }\end{array}$ \\
\hline
\end{tabular}




\begin{tabular}{|c|c|}
\hline & $\begin{array}{c}\text { - Le souci de la performance du fournisseur doit être gardé à } \\
\text { l'esprit tout au long la relation : Sélection, finalisation, pilotage } \\
\text { et post-évaluation. }\end{array}$ \\
\hline
\end{tabular}

Source : élaboration personnelles

\section{Tableau 5. Bonnes pratiques managériales (suite)}

\begin{tabular}{|c|c|c|}
\hline $\begin{array}{c}\text { Leviers } \\
\text { d'amélioration }\end{array}$ & $\begin{array}{c}\text { Bonnes pratiques } \\
\text { Les canaux de } \\
\text { communication }\end{array}$ & $\begin{array}{c}\text { - Programmer des journées fournisseurs et des comités de pilotage } \\
\text { pour communiquer sur les objectifs, les résultats, les nouveaux } \\
\text { projets, etc. ; }\end{array}$ \\
\hline - Accompagner le fournisseur dans sa démarche de mise en place \\
de l'échange informatisé EDI ; \\
- Assurer un échange régulier entre les acteurs sous différentes \\
formes : réunions, téléphones, visioconférences, emails ; \\
- Mettre à la disposition des fournisseurs toute la documentation \\
nécessaire.
\end{tabular}

\section{Conclusion}

Source : élaboration personnelles

Cette étude a mis en évidence le processus de pilotage de la performance des fournisseurs par les pratiques de SCM, et elle nous a permis d'intégrer l'audit combiné "qualité et logistique» comme une pratique innovante dans ce système.

Nos résultats permettent d'apporter une contribution à la connaissance théorique et pratique relatives à la gestion des fournisseurs, à travers le processus de pilotage de la performance, dans un secteur d'activité très riche 
en bonnes pratiques en gestion. Ainsi, dans l'analyse des résultats, nous avons tiré une panoplie des bonnes pratiques qui peuvent être d'une grande utilité pour les managers.

Les résultats et les renseignements auxquels nous sommes parvenus dans le cadre de cette recherche sur le pilotage de la performance des fournisseurs, par une approche qualitative basée sur l'étude de cas, ont une forte validité interne au détriment d'une validité externe.

De plus, le caractère subjectif de la recherche limite la portée des conclusions. Ce caractère a été accepté comme un composant à part entière de la recherche. Dans cette recherche qualitative, le mode de généralisation théorique ne peut pas être statistique ; il est analytique (Yin, 1994). Notre étude repose sur l'étude d'un cas unique dans un contexte donné. En conséquence, on ne peut pas prétendre que notre modèle ainsi que les résultats présentés soient applicables d'une façon générale.

Dans cette recherche, nous avons analysé le phénomène de pilotage de la performance des fournisseurs et de la position d'un client donneur d'ordre, et nous n'avons intégré l'avis et la position des fournisseurs. Ce choix méthodologique ne permet pas de cerner ce sujet dans sa globalité. De même, le choix d'un seul secteur d'activité limite la validité externe de l'étude.

Pour cette raison, il serait intéressant d'élargir le champ d'étude aux sphères des fournisseurs afin de renforcer la généralisation analytique. Etudier l'impact des pratiques de SCM, du point de vue des fournisseurs, permettrait d'avoir une vision globale sur le phénomène et obtenir une liste des pratiques efficaces et acceptables par toutes les parties. Une seconde voie consiste à mener cette recherche auprès d'un autre constructeur automobile qui opère dans le contexte marocain, notamment PSA Peugeot Citroën, dont la production va démarrer en 2020. Une troisième voie consiste à chercher une validation externe à travers une enquête qualitative permettant de peaufiner et d'exploiter les pistes proposées. Cela permettra aussi de valider les résultats dans des contextes et des secteurs différents.

\section{References:}

1. Aguezzoul, A. \& Ladet, P. (2009). «Sélection et évaluation des fournisseurs: critères et méthodes », Revue Française de gestion industrielle, 1-21.

2. Anas Hattabou (2011). « Le pilotage de la performance globale entre logique de conformation et logique d'innovation : une approche par les systèmes Agoantagonistes - cas de deux entreprises pionnières au 
Maroc-», thèse de doctorat en sciences de gestion, université cadi Ayyad, Marrakech.

3. Boissinot, A. (2008). « Le management des prestataires : vers une approche personnalisée de la relation ? ", XVIIème Conférence Internationale de Management Stratégique, Nice Sofia Antipolis.

4. Brulhart, F. \& Moncef, B. (2009). « Supply Chain Management et performance : quel impact des pratiques de SCM sur la création de valeur ? », Finance Contrôle Stratégie - volume 13, n 1, mars 2010, p. 33-66.

5. Chen, I.J. \& Paulraj, A. (2004). "Towards a theory of supply chain management: the constructs and measurements". Journal of Operations Management 22 (2), 119-150.

6. Chen, Y.-J., (211). "Structured methodology for supplier selection and evaluation in a supply chain".Inf.Sci.181 (9), 1651-1670.

7. Chow, W., Madu, C., Kuei, C., Lu, M., Lin, C., \& Tseng, H. (2008). "Supply chain management in the us and Taiwan: an empirical study". Omega 36 (5), 665-679.

8. Donada, C. (1997). « Fournisseurs : pour déjouer les pièges du partenariat », Revue Française de Gestion, $n^{\circ} 114$, juin-juillet-août, pp. 94-105.

9. Douglas, ML., Keely, L., Croxton Sebastián, J., \& García-Dastugue (2001). "The Supply Chain Management Processes", The International Journal of Logistics Management, Vol. 12, No. 2, pp. 2-36.

10. Durand, B. \& Faultrier, B. (2007). «L'impact de la supplychain sur les métiers de la logistique et des achats" Logistique \& Management, Volume 15, Number 2, 2007, pp. 55-70(16)

11. Flynn, B. \& Flynn, E. (2005). «Synergies between supply chain management and quality management: emerging implications», International Journal of Production Research, vol. 43, no. 16, pp. 34213436.

12. Guibert, N. (1996). "La relation client-fournisseur et les nouvelles technologies de l'information : le rôle des concepts de confiance et d'engagement $»$. Thèse pour l'obtention du titre de docteur ès sciences de gestion, Université de Montpellier II.

13. Hohmann, C. (2004). «Audit combiné : qualité/supplychain ». Edition d'organisation.

14. Hsu, C.-C., Tan, K.C., Kannan, V.R., \& Leong Keong, G. (2009). "Supply chain management practices as a mediator of the relationship 
between operations capability and firm performance ", International Journal of Production Research, 47:3, 835-855.

15. Jabbour, A. \& Jabbour, C. (2009). «Are supplier selection criteria going green? Case studies of companies in Brazil », Industrial Management \& Data Systems, Vol. 109 Issue: 4, pp.477-495.

16. Jean-Jacques Laville (2006). « Comment sécuriser sa supply chain?», Logistique \& Management, Vol. $14-\mathrm{N}^{\circ} 1,3-18$.

17. Jenn Olivier (2008). "Outils de gestion du "risque fournisseur " : méthodes et modèles pour la sélection des fournisseurs » Mémoire. Montréal (Québec, Canada), Université du Québec à Montréal, Maîtrise en administration des affaires.

18. Jobin, MH. \& Friel, T. (2001). «Sur la piste de la création durable de valeur à travers la chaîne logistique », Logistique \& Management, Numéro : 17 - Vol.9 n².

19. Koh, S., Demirbag, M., Bayraktar, E., Tatoglu, E., \& Zaim, S. (2007). "The impact of supply chain management practices on performance of SMEs". Industrial Management and Data Systems 107 (1), 103.

20. Kotabe, M., Martin, X., \& Domoto, H. (2003). " Gaining from vertical partnerships: knowledge transfer, relationship duration, and supplier performance improvement in the U.S. and Japanese automotive industries". Strategic Management Journal 24, 293-316.

21. Li, S., Ragu-Nathan, B., Ragu-Nathan, T.S., \& Rao, S.S. (2006). "The impact of supply chain management practices on competitive advantage and organizational performance”. Omega 34 (2), 107-124.

22. Min, S. \& Mentzer, J. (2004). "Developing and measuring supply chain management concepts". Journal of Business Logistics 25 (1), 63-100.

23. Nogatchewsky, G. (2004). «Les configurations de contrôle dans les relations client-fournisseur» Thèse de doctorat en sciences de gestion. Université Paris Dauphine.

24. Park, J., Shin, D., Chang, T.W., \& Park (2010). "An integrative framework for supplier relationship management".Ind.Manag.DataSyst.110 (4), 495-515.

25. Paul Cousins, Benn Lawson, \& Brian Squire (2008). "Performance measurement in strategic buyer-supplier relationships: The mediating 
role of socialization mechanisms", International Journal of Operations \& Production Management, Vol. 28 Iss: 3 pp. $238-258$

26. Paulraj, A. \& Chen, I.J. (2004). "Understanding supply chain management: critical research and a theoretical framework". International Journal of Production Research vol. 42, no. 1.

27. Salgado, M. (2013). «La performance : une dimension fondamentale pour l'évaluation des entreprises et des organisations», <hal00842219>, 1-10.

28. Sharma, V., Sahay, B.S., \& Sardana, G.D. (2008). «An Empirical Assessment of the Impact of SCM Practices on Quality Performance: A Case in the Indian Automobile Industry », Supply Chain Forum An International Journal, Vol. 9 - $\mathrm{N}^{\circ} 1$-pp 28-40.

29. Shin, H., Collier, D.A., \& Wilson, D.D. (2000). "Supply management orientation and supplier/buyer performance", Journal of Operations Management, Vol. 8, pp. 317-33.

30. Silva, E.M., Neto, M.S., \& Pires, S.R.I. (2012). «An evaluation of the variables and terminologies employed in the constructs of SCM practices » ISSN: 1984-3046 - Journal of Operations and Supply Chain Management 5 (1), pp 1 - 15.

31. Souilah, S. (2009). « Reengineering du pilotage des flux dans une relation client/fournisseur : application au cas de l'industrie automobile », thèse de doctorat, Ecole centrale des arts et « Ecole centrale Paris».

32. Suhong Li, Bhanu Ragu-Nathan, Ragu-Nathan, T.S., \& Subba Rao, S. (2006). "The impact of supply chain management practices on competitive advantage and organizational performance", International Journal of management science Omega 34, p. 107 - 124.

33. Suhong Li, Ragu-Nathan, T. S., \& Subba Rao (2005). « Development and Validation of a Measurement Instrument for Studying Supply Chain Practices ", journal of operations management $\cdot$ septembre 2005.

34. Tan, K.C., Lyman, S.B., \& Wisner, J.D. (2002). "Supply chain management: a strategic perspective". International Journal of Operations \& Productions Management 22 (6), 614-631.

35. Tan, K.C., Lyman, S.B., \& Wisner, J.D. (2002). "Supply chain management: a strategic perspective". International Journal of Operations \& Productions Management 22 (6), 614-631.

36. Vanichchinchai, A. \& Igel, B. (2011). «The impact of total quality management on supply chain management and firm's supply 
performance », International Journal of Production Research, Vol. 49, No. 11, 1 June 2011, 3405-3424.

37. Yin, R.K. (1984). «Case Study Research: Design and Methods», Sage Publications, eds. 2002. 